\title{
Context Is Paramount in the Assessment of Meta-analytical Evidence: Reply
}

\author{
Emmanouil Zacharakis • Babar Kayani • \\ Thanos Athanasiou $\cdot$ Hutan Ashrafian
}

Published online: 1 December 2012

(C) Société Internationale de Chirurgie 2012

We appreciate Cavallin et al. [1] commenting on our manuscript. They remark that our study selection demonstrates heterogeneity in body mass index (BMI) groups with regard to (1) clinical outcomes and (2) disease pathology, emphasizing these limitations as "weak points."

In our study we aimed at deriving the best possible evidence from the available studies in the literature to inform clinical practice. As a result, we applied the internationally accepted World Health Organization classification for categorizing obese and non obese patients according to body mass index above and below $30 \mathrm{~kg} / \mathrm{m}^{2}$. Although this division is characterized by some heterogeneity in clinical parameters, it offered us the opportunity to synthesize data from the available literature to derive some clinical value. This was discussed in depth in our limitations section, demonstrating transparency and openness to criticism.

Our statistical analysis was based on a random effects model so that all our results were derived from the most conservative estimates in order to minimize any overestimation of effect sizes. Furthermore all our reported results demonstrate very low levels of statistical heterogeneity, which adds support to the robustness of these preliminary findings.

As a result, we feel that our manuscript deserves consideration within the contextual application of evidence [2]. As we have already stated in our conclusion, we emphasized the need for further studies in this area rather than proposing new recommendations. Consequently we feel that this work may be informative to readers of the journal and may guide future clinical research directions.

\section{References}

1. Cavallin F, Scarpa M, Cagol M et al (2012) Does obesity affect outcomes in patients undergoing esophagectomy for cancer? Comments on a meta-analysis. World J Surg 36:1785-1795. doi: 10.1007/s00268-012-1800-0

2. Greenhalgh $T$, Wieringa $S$ (2011) Is it time to drop the "knowledge translation" metaphor? A critical literature review. J R Soc Med 104:501-509
E. Zacharakis $(\bowtie) \cdot$ B. Kayani - T. Athanasiou - H. Ashrafian Department of Surgery and Cancer, Imperial College London, 10th Floor, QEQM Wing, St. Mary's Campus,

London W2 1NY, UK

e-mail: manoszacharakis@hotmail.com 